\title{
Route to achieving perfect $B$-site ordering in double perovskite thin films
}

\author{
Josée E Kleibeuker ${ }^{1}$, Eun-Mi Choi ${ }^{1}$, Edward D Jones ${ }^{1}$, Tse-Min Yu $^{2}$, Bianca Sala ${ }^{2}$, Belinda A MacLaren ${ }^{2}$, \\ Demie Kepaptsoglou ${ }^{3}$, David Hernandez-Maldonado ${ }^{3}$, Quentin M Ramasse ${ }^{3}$, Lewys Jones ${ }^{4}$, Juri Barthel ${ }^{5}$, \\ Ian MacLaren ${ }^{2}$ and Judith L MacManus-Driscoll ${ }^{1}$
}

Double perovskites (DP, $A_{2} B B^{\prime} \mathrm{O}_{6}$ ) exhibit a breadth of multifunctional properties with a huge potential range of applications, including magneto-optic and spintronic devices. However, spontaneous cation ordering is limited by the similar size and charge of $B$ and $B^{\prime}$ cations. We introduce a route to stimulate $B$-site rock-salt ordering. By growing thin films on (111)-oriented substrates, 'in-plane' strain acts on the intrinsically tilted oxygen octahedra of the DP and produces two different $B$-site cages (in size and shape), stimulating spontaneous cation ordering. For the ferromagnetic insulator $\mathrm{La}_{2} \mathrm{CoMnO}_{6}, \mathrm{clear} \mathrm{Co} / \mathrm{Mn}$ ordering was achieved by growing on (111)-oriented substrates. The difference in $B$-site cages was further enhanced when grown under minor (111) in-plane compressive strain, resulting in long-range ordering with a saturation magnetization of $5.8 \mu_{B} /$ formula unit (f.u.), close to the theoretical $6 \mu_{\mathrm{B}} /$ f.u., without antiferromagnetic behavior. Our approach enables the study of many new ordered DPs which have never been made before.

NPG Asia Materials (2017) 9, e406; doi:10.1038/am.2017.113; published online 14 July 2017

\section{INTRODUCTION}

Bi-component perovskites, $A A^{\prime} B B^{\prime} \mathrm{O}_{6}$, both artificially grown as ultrashort period perovskite-oxide superlattices and as spontaneously ordered double perovskites (DP), attract high interest due to their potential application in a wide range of devices, such as electrically tunable microwave devices, ${ }^{1}$ multiferroic solar cells, ${ }^{2}$ magneto-optic devices $^{3}$ and spin-filter devices. ${ }^{4}$ In this materials family, the cations are ordered in either a layered, columnar or rock salt structure. As a result, these materials exhibit a large set of exotic magnetic and ferroelectric properties that are absent in the parent compounds, including improper ferroelectricity (for example, in $\mathrm{PbSrTi}_{2} \mathrm{O}_{6}$ ), ${ }^{5}$ strongly enhanced dielectric constant (for example, in $\mathrm{BaSrTi}_{2} \mathrm{O}_{6}$ ), ${ }^{6}$ ferromagnetic semiconducting behavior (for example, in $\mathrm{La}_{2} \mathrm{NiMnO}_{6}$ ), ${ }^{7}$ multiferroicity (for example, in $\left.\mathrm{Bi}_{2} \mathrm{NiMnO}_{6}\right)^{8}$ and room temperature colossal magnetoresistance (for example, in $\mathrm{Sr}_{2} \mathrm{Fe}$ $\left.\mathrm{MoO}_{6}\right) .{ }^{9}$ In addition, there are many experimentally unexplored bi-component perovskites that are suggested to have fascinating properties. Examples are hybrid improper ferroelectric $\mathrm{LaYGa}_{2} \mathrm{O}_{6},{ }^{10}$ low-band-gap $\mathrm{Sr}_{2} \mathrm{AlVO}_{6}$, ${ }^{11}$ half-metallic ferromagnet $\mathrm{Sr}_{2} \mathrm{TiCrO}_{6}{ }^{12}$ and half-metallic antiferromagnet $\mathrm{La}_{2} \mathrm{VCuO}_{6} \cdot{ }^{13}$ However, to achieve these exciting functionalities, the materials should have a high degree of cation ordering. Unfortunately, the fabrication of ultrashort period superlattices is extremely difficult and a slow growth rate is required, limiting industrial interest. Therefore, to enable the fabrication of bi- component perovskite thin films with perfect ordering and thus realizing their huge potential, one has to look for routes that allow natural formation of these superlattices, that is, fabrication of DP thin films.

Considering DP $B$-site cation ordering in bulk, the biggest challenge is the similarity of the ionic formal valence $(\mathrm{FV})$ and ionic radius $\left(r_{B}\right)$ of the $B$-site cations. Hence, to achieve spontaneous $B$-site rock salt ordering in bulk, a distinct difference in FV and $r_{B}$ is necessary. ${ }^{14,15}$ Similar constraints apply for thin films, but, in addition, thin film growth is complicated by the presence of multiple multivalent ions in DPs. ${ }^{16,17}$ These constraints limit the group of ordered DP thin films significantly, and therefore, to date, only a small number of ordered DP thin films has been fabricated. On the other hand, thin film growth offers an additional set of growth parameters to play with, including anisotropic strain. Optimizing these growth parameters has already shown to enable the growth of spontaneously ordered DP thin film materials that do not exist in bulk. ${ }^{18-22}$

Promisingly, for example, recent studies have shown that control of thin film growth kinetics can lead to spontaneous ordering even if the difference in FV and $r_{B}$ is negligible. ${ }^{18-22}$ Here, cation ordering can be stimulated by using non-equilibrium growth settings during pulsed laser deposition, that is, a low oxygen pressure $\left(\sim 10^{-4} \mathrm{mbar}\right)$, high substrate temperature $\left(>1000^{\circ} \mathrm{C}\right)$ and subsequent quenching to room temperature. It was proposed that ordering may occur under these

\footnotetext{
${ }^{1}$ Department of Materials Science and Metallurgy, University of Cambridge, Cambridge, UK; ${ }^{2}$ SUPA School of Physics and Astronomy, University of Glasgow, Glasgow, UK; ${ }^{3}$ SuperSTEM Laboratory, STFC Daresbury Laboratory, Daresbury, UK; ${ }^{4}$ Department of Materials, University of Oxford, Oxford, UK and ${ }^{5}$ Ernst Ruska-Centre (ER-C) for Microscopy and Spectroscopy with Electrons, Forschungszentrum Jülich GmbH, Jülich, Germany

Correspondence: Professor JL MacManus-Driscoll, Department of Materials Science and Metallurgy, University of Cambridge, 27 Charles Babbage Road, Cambridge CB3 OFS, UK.

E-mail: jld35@cam.ac.uk

Received 31 December 2016; revised 31 March 2017; accepted 9 May 2017
} 
a

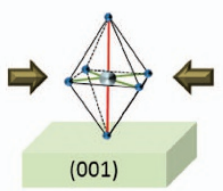

c

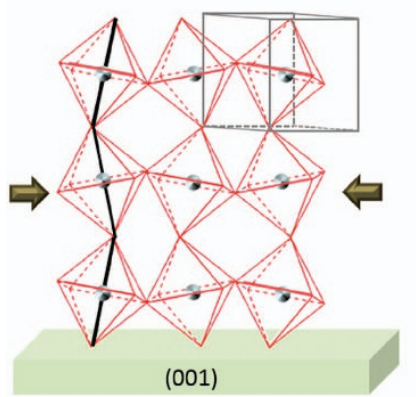

b

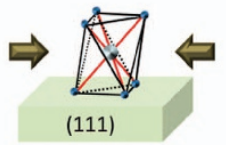

d

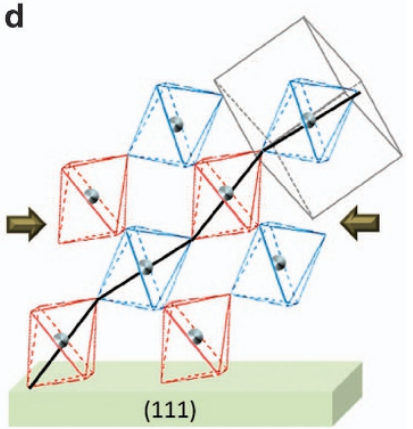

Figure 1 The effect of compressive in-plane strain on the $\mathrm{BO}_{6}$ octahedra of a cubic $\mathrm{ABO}_{3}$ structure on substrates with (001)-orientation (a) and (111)orientation (b), where the green lines indicate a reduced $d_{B-0}$ and the red lines indicate an elongated $d_{B-0}$ compared to the non-strained $B O_{6}$. Schematic drawings of the oxygen octahedra in perovskite films that contain anti-phase rotations after applying in-plane compressive strain are shown in (c and d); (c) for (001)-oriented films and (d) for (111)-oriented films. The oxygen ions are at the corners of the octahedra and the $B$ cations are at the center of the octahedra. The $A$-site cations are not depicted but would lie in the open spaces between the octahedra. The cubes depict the pseudocubic $\mathrm{ABO}_{3}$ unit cell. The two different octahedra formed in a film on the (111)oriented substrate are depicted in blue and red. The zig-zag pattern of the $-0-B-O-B$ - chain is highlighted in black.

conditions as the FV difference between $B$ and $B$ ' is enhanced in the surface growth front. ${ }^{22}$ However, the exact understanding of why $B$ site ordering takes place was not made clear. Also, the complexity of the growth and reproducibility of the growth remain challenging. In addition, the required high substrate temperature and low pressure during growth are not suitable for many DP compositions, including $\mathrm{Sr}_{2} \mathrm{FeMoO}_{6}, \mathrm{La}_{2} \mathrm{CoMnO}_{6}$ and the highly demanding multiferroic $\mathrm{Pb}$ - or Bi-containing DPs. ${ }^{8,23-25}$

Therefore, understanding of what leads to spontaneous $B$-site ordering in thin films and developing new growth processes that produce this ordering reliably are now required. Here, we start with what is known for bulk $A$-site ordering, where the presence of two differently sized spaces at the $A$-sites, as a result of $a^{+} a^{+} a^{+} B_{6}$ octahedral tilting (Glazer's notation ${ }^{26}$ ), produces spontaneous $A$-site cation ordering in the highly complex $A_{3} A^{\prime} B_{4} \mathrm{O}_{12}$ system. $^{27}$ Transferring this route to the $B$-sites in $A_{2} B B^{\prime} O_{6}$ thin films would require the formation of different $B$-site cages (in shape and volume) in an ordered arrangement. Analogously to the scenario described for the $A$-sites, we propose here that two different spaces at the $B$-site can be created in a rock salt ordered manner when the film material contains anti-phase octahedral rotations in combination with induced 'in-plane' strain, which is controlled by the orientation of the substrate and the substrate-film misfit. We investigate experimentally whether this approach can enhance or even induce spontaneous $B$-site ordering.

To understand how two different $B$-sites can be created, we first discuss the effect of 'in-plane' strain on non-distorted $\mathrm{BO}_{6}$ for different substrate orientations (see Figures $1 \mathrm{a}$ and $\mathrm{b}$ ). First of all, for the most conventionally used orientation, the (001), a perovskite under compressive in-plane strain has four reduced in-plane $B$-O bonds $\left(d_{B-\mathrm{O}}\right)$ and two elongated out-of-plane $d_{B-\mathrm{O}}$ (Figure 1a). On the other hand, for the rarely studied (111)-oriented perovskites under compressive strain, all six $d_{B-O}$ elongate to preserve the unit cell volume. (Figure 1b)

Secondly, we include octahedral rotations, as they are present in the majority of DP-oxides. ${ }^{28}$ The rotations of the octahedra result in a zig-zag pattern of the -O- $B-\mathrm{O}-B$ - chain, as depicted in Figures $1 \mathrm{C}$ and d. For (001)-oriented films, the rotation axes are either parallel or perpendicular to the substrate surface (Figure 1c). Therefore, although the octahedra are tilted, the effect of strain is expected to be the same on all tilted $\mathrm{BO}_{6}$ octahedra, independent of the type of rotation (in-phase or anti-phase). For (111)-oriented films, one set of $\mathrm{BO}_{6}$ octahedra tilts towards the substrate surface plane (the in-plane direction), while the nearest neighboring set of $\mathrm{BO}_{6}$ octahedra tilts toward the substrate surface normal (the out-of-plane direction) as illustrated in Figure 1d. Hence, the effect of in-plane strain on the two sets of octahedra is different, resulting in $B$-site cages of different orientation and volume. The difference between the octahedra depends on the magnitude of the strain and the magnitude of the octahedral tilting. A typical volume difference between the two octahedra would be between $0.3-3 \%$. Both octahedra are tilted similar with respect to the out-of-plane direction. Therefore, applying in-plane strain on these octahedra results in a shape and volume change that is the same for both octahedra. Note that cation displacements are not taken into account. Since the two different $B$-site cages are nearest neighbors, they are then arranged in a rock salt fashion, which is the most frequently found arrangement of $B$ cations in ordered $A_{2} B B^{\prime} \mathrm{O}_{6} \cdot{ }^{15}$ Having small differences between $B$ and $B$, such as in valence state, ionic radius and electronegativity, is expected to stimulate an ordered occupation of the $B$-site cages instead of forming a random arrangement.

To test the above hypothesis about the formation of two different $B$-site cages stimulating $B$-site cation ordering, we have grown the ferromagnetic insulator $\mathrm{La}_{2} \mathrm{CoMnO}_{6}$ (monoclinic space group $\mathrm{P}_{1} / n$, $a_{\text {monoclinic }}=0.553 \mathrm{~nm}, \quad b_{\text {monoclinic }}=0.549 \mathrm{~nm}, \quad c_{\text {monoclinic }}=0.778 \mathrm{~nm}$ and $\beta_{\text {monoclinic }}=89.95^{\circ}$ giving $a_{\mathrm{pc}}=0.389 \mathrm{~nm}$, where $\mathrm{pc}$ denotes pseudocubic) on various (001)- and (111)-oriented substrates with different strain states (see Figure 2a). ${ }^{29}$

$\mathrm{La}_{2} \mathrm{CoMnO}_{6}$ is used here as a model system, since it offers some significant advantages. First of all, bulk $\mathrm{La}_{2} \mathrm{CoMnO}_{6}$ has an $a^{-} a^{-} c^{+}$ tilting pattern (Glazer's notation ${ }^{26}$ ). Having rotations along all three orthogonal axis of which two rotations are anti-phase, a clear difference in $B$-site space as a result of strain can be expected. (Note that the volumes of $\mathrm{MnO}_{6}$ and $\mathrm{CoO}_{6}$ in bulk ordered $\mathrm{La}_{2} \mathrm{CoMnO}_{6}$ have a difference of $\sim 13 \%{ }^{29}$ ) Secondly, it is a challenge to achieve full $\mathrm{Co} / \mathrm{Mn}$ ordering as both ordered and disordered $\mathrm{La}_{2} \mathrm{CoMnO}_{6}$ exist in bulk. Ordered $\mathrm{La}_{2} \mathrm{CoMnO}_{6}$ can occur as a result of internal charge transfer from $\mathrm{Mn}$ to $\mathrm{Co}$, giving $\mathrm{Mn}^{4+}$ and $\mathrm{Co}^{2+}\left(\Delta \mathrm{FV}=2\right.$ and $\left.\Delta \mathrm{r}_{\mathrm{B}}=11.5 \mathrm{pm}\right){ }^{29-31}$ However, disordered $\mathrm{La}_{2} \mathrm{CoMnO}_{6}$ contains $\mathrm{Co}^{3+} / \mathrm{Mn}^{3+}\left(\Delta \mathrm{FV}=0\right.$ and $\left.\Delta \mathrm{r}_{\mathrm{B}}=3.5 \mathrm{pm}\right)$ and is energetically $(0.2 \mathrm{eV})$ slightly more favorable than $\mathrm{Co}^{2+} / \mathrm{Mn}^{4+} \cdot{ }^{29,30,32}$ As a result, a very high degree of $\mathrm{Co} / \mathrm{Mn}$ ordering in thin films has not been achieved to date. Using the formation of two different $B$-site spaces as an extra driving force to create $\mathrm{Co} / \mathrm{Mn}$ ordering, we expect that highly ordered $\mathrm{La}_{2} \mathrm{CoMnO}_{6}$ films can be achieved on (111)-oriented substrates. Since this driving force is absent for films on (001)-oriented substrates, a low degree of cation ordering is expected in these cases.

To date, achieving cation ordering in $\mathrm{La}_{2} \mathrm{CoMnO}_{6}$ in both bulk and thin films requires high growth temperatures (for example, $>1100{ }^{\circ} \mathrm{C}$ in bulk) as well as high oxygen pressures. ${ }^{33-35}$ As a result, the non-equilibrium DP thin film growth method, using low oxygen 
a

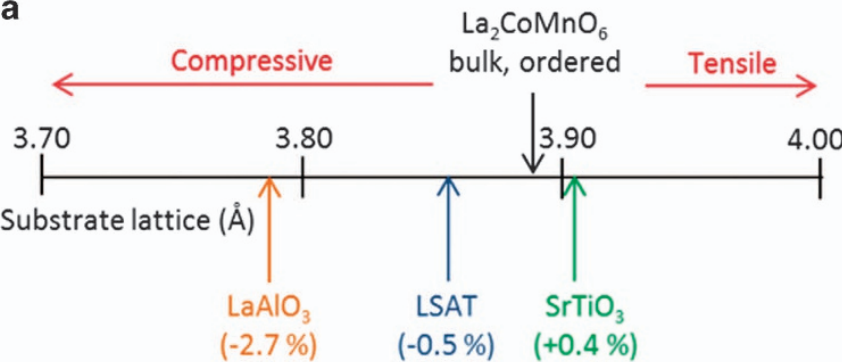

b

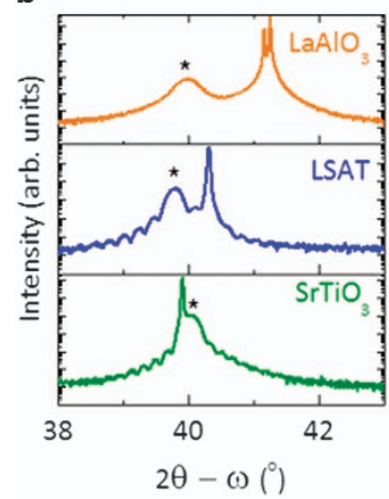

c

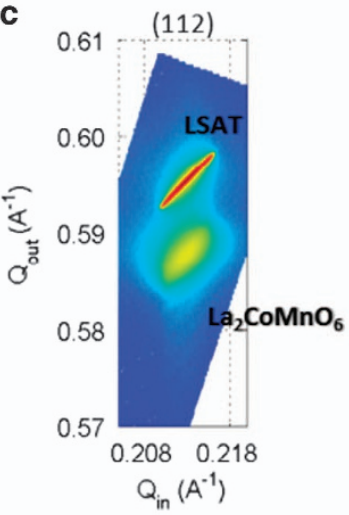

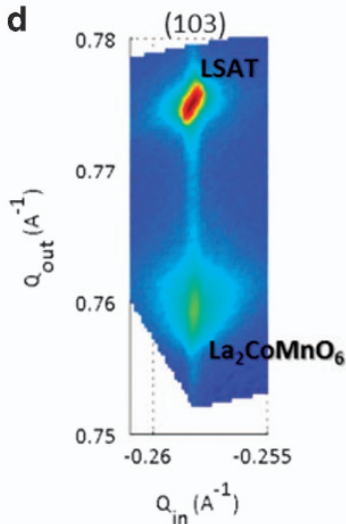

Figure 2 (a) The pseudocubic lattice parameters and mismatch of various perovskite substrates and $\mathrm{La}_{2} \mathrm{CoMnO}_{6}$. (b) XRD out-of-plane diffraction patterns around the $(111)_{\mathrm{pc}}$ of $\mathrm{La}_{2} \mathrm{CoMnO}_{6}$ films grown on the various substrates along the (111). The $\mathrm{La}_{2} \mathrm{CoMnO}_{6}$ peak is marked by $\left.{ }^{*}\right)$, the sharp intense peaks are from the substrate. (c and d) Reciprocal space maps of $\mathrm{La}_{2} \mathrm{CoMnO}_{6}$ grown on LSAT (111) and (001), respectively.

pressures, cannot be used for $\mathrm{La}_{2} \mathrm{CoMnO}_{6}$. In $\mathrm{La}_{2} \mathrm{CoMnO}_{6}$, low oxygen pressures lead to oxygen vacancies, which are associated with cation disorder. ${ }^{30}$ Additionally, the magnetic Curie temperature $\left(T_{\mathrm{C}}\right)$ and the saturation magnetization $\left(M_{S}\right)$ of $\mathrm{La}_{2} \mathrm{CoMnO}_{6}$ strongly depend on the cation ordering, making the magnetic behavior a good indicator for the presence of cation ordering. ${ }^{17,36}$ Bulk ordered $\mathrm{La}_{2} \mathrm{Co}^{2+} \mathrm{Mn}^{4+} \mathrm{O}_{6}$ has a ferromagnetic-to-paramagnetic transition temperature $\left(T_{\mathrm{C}}\right)$ of $\sim 230 \mathrm{~K}$, while a $T_{\mathrm{C}}$ of $\sim 80-150 \mathrm{~K}$ is often related to the disordered $\mathrm{La}_{2} \mathrm{Co}^{3+} \mathrm{Mn}^{3+} \mathrm{O}_{6}$ phase. ${ }^{30,36}$ Furthermore, fully ordered $\mathrm{La}_{2} \mathrm{CoMnO}_{6}$ has a theoretical spin-only $M_{s}$ of $6 \mu_{\mathrm{B}}$ /formula unit (f.u.). ${ }^{30}$ The introduction of disorder at the $B$-site as a result of antisites or anti-phase boundaries leads to antiferromagnetic (AFM) $\mathrm{Co}^{2+}-\mathrm{O}-\mathrm{Co}^{2+}$ and $\mathrm{Mn}^{4+}-\mathrm{O}-\mathrm{Mn}^{4+}$ superexchange interactions, lowering both $M_{s}$ and $T_{\mathrm{C}}{ }^{30}$ Moreover, the $T_{\mathrm{C}}$ and $M_{s}$ decrease by the introduction of oxygen vacancies arising from the formation of $\mathrm{Mn}^{3+}$ which lowers the degree of ordering. ${ }^{17,30,37}$

To study the influence of both the magnitude of strain and the substrate orientation, thin $\mathrm{La}_{2} \mathrm{CoMnO}_{6}$ films were grown by pulsed laser deposition on three different perovskite-oxide substrates $\left(\mathrm{LaAlO}_{3},(\mathrm{La}, \mathrm{Sr})(\mathrm{Al}, \mathrm{Ta}) \mathrm{O}_{3}\right.$ (LSAT) and $\left.\mathrm{SrTiO}_{3}\right)$ with two different orientations, either (001) or (111). The mismatch between substrate and ordered $\mathrm{La}_{2} \mathrm{CoMnO}_{6}$ is depicted in Figure 2a. To avoid any influence of the growth parameters on cation ordering, all films were grown under the same growth conditions. The growth settings were optimized for films on (111)-oriented $\mathrm{SrTiO}_{3}$ to achieve coherently-grown, single-phase $\mathrm{La}_{2} \mathrm{CoMnO}_{6}$. To minimize the effect of spontaneous bulk ordering, we used a growth temperature which was $50-100{ }^{\circ} \mathrm{C}$ lower than in previous investigations of $\mathrm{La}_{2} \mathrm{CoMnO}_{6}$ film growth. ${ }^{17,31,33,34,36,38-40}$ The films were deposited in the thickness range of $20-60 \mathrm{~nm}$, which is sufficiently low to allow only minimal in-plane relaxations on the $\mathrm{SrTiO}_{3}$ and LSAT substrates, but not too low to result in a $T_{\mathrm{C}}$ reduction. ${ }^{41-43}$

\section{METHODS}

\section{Target fabrication}

A ceramic $\mathrm{La}_{2} \mathrm{CoMnO}_{6}$ target was synthesized by conventional solid-state reaction. A stoichiometric mixture of $\mathrm{Co}_{3} \mathrm{O}_{4}$ (Alfa Aesar, Lancashire, UK), $\mathrm{MnO}_{2}$ (Alfa Aesar) and $\mathrm{La}_{2} \mathrm{O}_{3}$ (Sigma Aldrich, Dorset, UK) was ground and subsequently calcined at $900{ }^{\circ} \mathrm{C}$ for $10 \mathrm{~h}$ under oxygen flow. After cool down the mixture was ground and pressed into a 1 inch pellet. The pellet was sintered at $1200{ }^{\circ} \mathrm{C}$ for $17 \mathrm{~h}$ under oxygen flow. Single-phase $\mathrm{La}(\mathrm{Co}, \mathrm{Mn}) \mathrm{O}_{3}$ was confirmed by powder X-ray diffraction (XRD, Bruker D8 theta/theta diffractometer, $\mathrm{Cu} \mathrm{K} \alpha$ radiation (Bruker AXS GmbH, Karlsruhe, Germay)).

\section{Thin film fabrication}

As substrates, we used $\mathrm{TiO}_{2}$ terminated (001)-and (111)-oriented $\mathrm{SrTiO}_{3}$ $(a=0.3905 \mathrm{~nm}),{ }^{44,45}$ thermally treated (001)-and (111)-oriented LSAT $(a=0.387 \mathrm{~nm})^{46}$ and thermally treated (111)-oriented $\mathrm{LaAlO}_{3}$ $\left(a_{\mathrm{pc}}=0.382 \mathrm{~nm}\right)^{47}$ single-crystal substrates (Crystec GmbH, Berlin, Germany). The films were grown by pulsed laser deposition at a substrate temperature of $710{ }^{\circ} \mathrm{C}$ with a repetition rate of $1 \mathrm{~Hz}$ and a fluence of $1.5 \mathrm{~J} \mathrm{~cm}^{-2}$. To ensure oxygen stoichiometry, the films were grown at 0.15 mbar flowing $\mathrm{O}_{2}$, annealed in $500 \mathrm{mbar}_{2}$ at $700{ }^{\circ} \mathrm{C}$ for $30 \mathrm{~min}$ directly after deposition and slowly cooled afterwards $\left(-5^{\circ} \mathrm{C} \mathrm{min}{ }^{-1}\right.$ to $400{ }^{\circ} \mathrm{C}$ followed by $-10^{\circ} \mathrm{C} \mathrm{min}^{-1}$ to room temperature) while maintaining $500 \mathrm{mbar}_{2}$ background pressure.

\section{Characterization of structure and physical properties}

Structural analysis of the films was done by XRD (high-resolution Panalytical Empyrean vertical diffractometer, $\mathrm{Cu} \mathrm{K} \alpha$ radiation, Panalytical, Kassel, Germany). Magnetic property measurements were performed using a superconducting quantum interference device (SQUID magnetometer, Quantum Design, San Diego, CA, USA), with a temperature range of $5-300 \mathrm{~K}$ and up to $4 \mathrm{~T}$.

\section{Scanning transmission electron microscopy}

Samples were prepared for scanning transmission electron microscopy (STEM) investigations by a focused ion beam lift-out procedure using a FEI Nova 

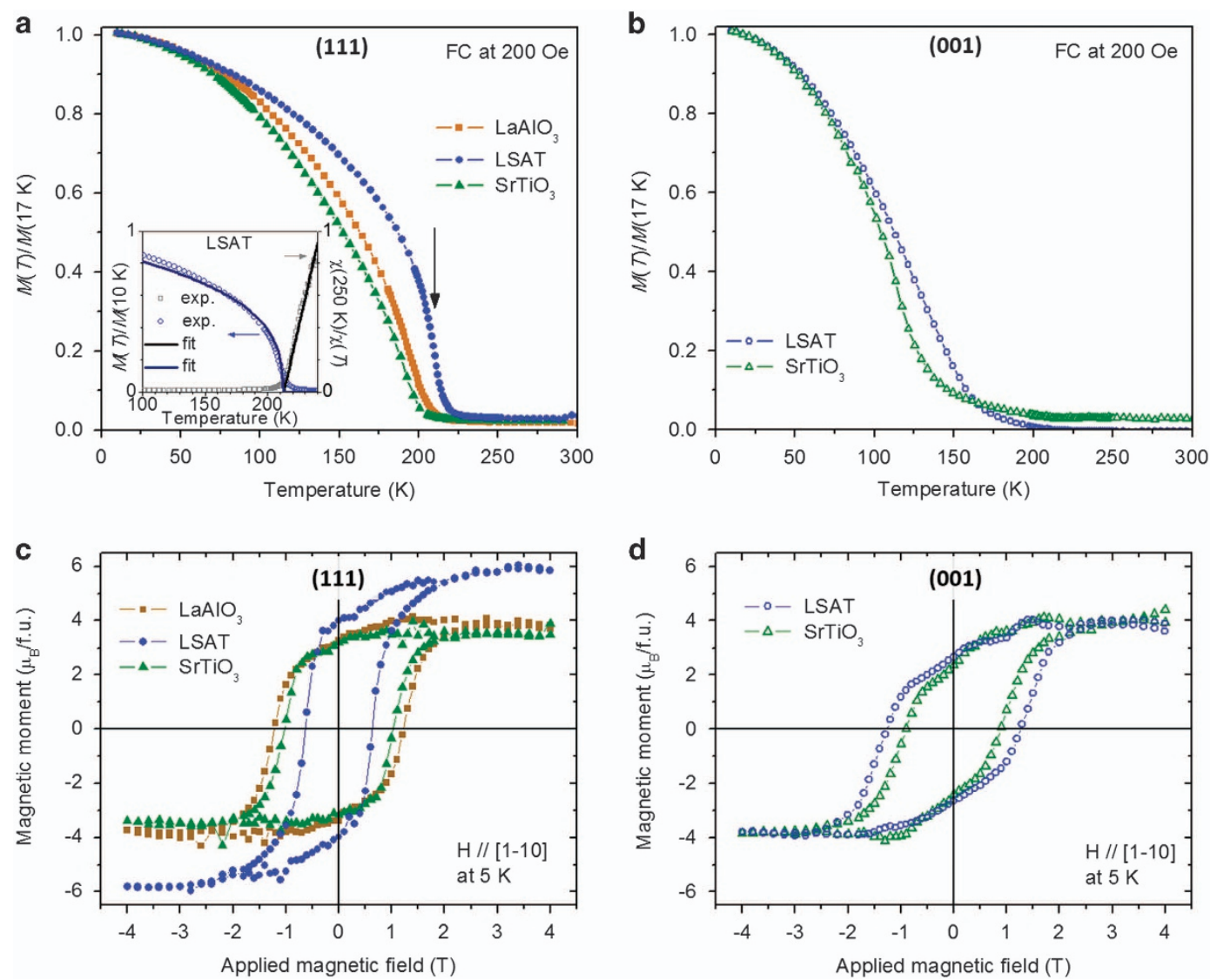

Figure 3 The normalized $M(T)$ data of the $\mathrm{La}_{2} \mathrm{CoMnO}_{6}$ films on (a) the (111)-oriented substrates and (b) the (001)-oriented substrates. The arrow in a marks the $T_{\mathrm{C}}$ of $\mathrm{La}_{2} \mathrm{CoMnO}_{6}$ on LSAT (111) at $211 \mathrm{~K}$. The inset of a shows normalized $M(T)$ and $\chi(T)$ of $\mathrm{La}_{2} \mathrm{CoMnO}_{6}$ on LSAT (111) near $T_{\mathrm{C}}$ as well as its corresponding Curie-Weiss fit. The in-plane $M(H)$ hysteresis curves of the corresponding films at $5 \mathrm{~K}$ are shown in $\mathbf{c}$ and $\mathbf{d}$. A linear paramagnetic background of the $\mathrm{SrTiO}_{3}$ substrate was subtracted.

Nanolab 200 DualBeam instrument (FEI, Hillsboro, OR, USA). Final polishing of the sections was performed using a $5 \mathrm{kV}$ Ga beam. In some cases, additional final polishing was performed using $500 \mathrm{~V} \mathrm{Ar}^{+}$ions in a Gatan PIPS (Gatan Inc., Pleasanton, CA, USA). The STEM measurements were performed using two instruments. Most of the data was recorded using a Nion UltraSTEM 100 (NION, Kirkland, WA, USA) operated at $100 \mathrm{kV}$ accelerating voltage with a probe semiconvergence angle of $30 \mathrm{mrad}$, and equipped with a Gatan Enfina Spectrometer with an acceptance semiangle of $32 \mathrm{mrad}$. High-angle annular dark field images were recorded with a detector with an effective inner angle of $\sim 78$ mrad. annular bright field (ABF) images were recorded with an angular range on the detector of 16-30 mrad, and simultaneously recorded low-angle annular dark field images were recorded with an angular range of 33-180 mrad. Additional data was recorded using a JEOL ARM200CF (JEOL Ltd., Akishima, Japan) operated at $200 \mathrm{kV}$ accelerating voltage and a probe convergence semiangle of $29 \mathrm{mrad}$, and equipped with a Gatan GIF Quantum ER with DualEELS using a $2.5 \mathrm{~mm}$ entrance aperture and $2 \mathrm{~cm}$ camera length to give an acceptance semiangle of $36 \mathrm{mrad}$. High-quality images were created by repeated scanning of the same area using a relatively short pixel dwell time of a few $\mu$ s, followed by subsequent alignment and summation, either using a simple rigid registration technique using the SDSD plugin for Digital Micrograph ${ }^{48}$ (Gatan Inc.) or using a non-rigid registration technique to also remove nonlinear scan-distortion and higher frequency electronic interference. ${ }^{49}$ Determination of atomic column positions was then performed using the Image Analysis routine for Digital Micrograph (kindly provided by Dr Bernhard Schaffer, Gatan $\mathrm{GmbH}$ ), followed by further analysis using conventional spreadsheets. Electron energy loss (EEL) spectrum images were processed by firstly removing noise using principal component analysis, ${ }^{50}$ followed by elemental mapping using Gatan Digital Micrograph. For the determination of oxidation states, standard spectra were acquired from samples of crushed powders of $\mathrm{CoO}\left(\mathrm{Co}^{2+}\right), \mathrm{LiCoO} 2\left(\mathrm{Co}^{3+}\right), \mathrm{Mn}_{2} \mathrm{O}_{3}\left(\mathrm{Mn}^{3+}\right)$ and $\mathrm{MnO}_{2}\left(\mathrm{Mn}^{4+}\right)$ (Sigma Aldrich Co.) under identical optical conditions to those used for the acquisition of the data from the $\mathrm{La}_{2} \mathrm{CoMnO}_{6}$ films, with both core-loss and low-loss data sets acquired from each area. As before, principal component analysis was used to reduce noise, and then the edge of interest was extracted by the fitting of an appropriate background before the edge. These were then transformed to a single-scattering distribution using Fourier-log deconvolution $^{51}$ to remove the effects of thickness and multiple scattering. A similar procedure was performed on real $\mathrm{Mn}$ and $\mathrm{Co}_{2,3}$ edges in the sample (where low-loss data were available for the deconvolution). Care needed to be taken with the extraction of the Co edges from the $\mathrm{La}_{2} \mathrm{CoMnO}_{6}$ samples, since the weak $\mathrm{Mn} \mathrm{L}_{1}$ edge lies just before the $\mathrm{Co}_{2,3}$ edge and the perturbation from this needed subtraction after background fitting. This then allowed the direct comparison of the real edges to those from standards.

\section{RESULTS AND DISCUSSION}

Structural analysis of the films was done by XRD. Figure $2 \mathrm{~b}$ shows the out-of-plane diffraction patterns of the films grown on the (111)-oriented substrates (reflections are indexed based on pseudocubic symmetry). The films were grown fully epitaxial and no impurity phases were observed. Thickness fringes for films on $\mathrm{SrTiO}_{3}$ and LSAT were clearly visible indicating high film quality with well-defined interfaces. No superlattice peaks as a result of cation ordering were observed along [111]. However, taking only cation ordering into account and no $A$-site displacement, these peaks are expected to be within the noise level due to the similar scattering factors of Co and Mn. ${ }^{18,52}$ 
Table 1 Overview of the magnetic results $\left(T_{\mathrm{C}}, M_{s}\right.$ and $\left.H_{\mathrm{c}}\right)$ of $\mathrm{La}_{2} \mathrm{CoMnO}_{6}$ on the various substrates

\begin{tabular}{|c|c|c|c|c|c|}
\hline Orientation & Substrate & Pseudocubic misfit (\%) & $T_{\mathrm{C}}(K)$ & $M_{s}\left(\mu_{\mathrm{B}} / f . u.\right)$ & $H_{c}(k O e)$ \\
\hline \multirow[t]{3}{*}{ (111) } & $\mathrm{LaAlO}_{3}$ & -2.7 & $193 \pm 4$ & 3.9 & 12 \\
\hline & LSAT & -0.5 & $211 \pm 1$ & 5.8 & 6 \\
\hline & $\mathrm{SrTiO}_{3}$ & +0.4 & $192 \pm 4$ & 3.5 & 10 \\
\hline \multirow[t]{2}{*}{ (001) } & LSAT & -0.5 & $125 \pm 8$ & 4.0 & 13 \\
\hline & $\mathrm{SrTiO}_{3}$ & +0.4 & $113 \pm 4$ & 3.9 & 9 \\
\hline
\end{tabular}

Asymmetric X-ray reciprocal space maps were taken to verify the strain state of the films (see Figures $2 \mathrm{c}$ and $\mathrm{d}$ for $\mathrm{La}_{2} \mathrm{CoMnO}_{6}$ on LSAT (111) and (001)). $\mathrm{La}_{2} \mathrm{CoMnO}_{6}$ on both $\mathrm{SrTiO}_{3}$ and LSAT substrates appeared to be fully in-plane strained, independent of the orientation. On the other hand, the misfit between $\mathrm{La}_{2} \mathrm{CoMnO}_{6}$ and $\mathrm{LaAlO}_{3}$ was too large $(-2.7 \%)$ to accommodate the stress in the film and, as a result, the film was fully relaxed. This result is consistent with the absence of thickness fringes in the out-of-plane diffraction pattern (see Figure 2b).

To verify the insulating state of the $\mathrm{La}_{2} \mathrm{CoMnO}_{6}$ films, four-point transport measurements for all samples were performed between 10-300 K. All samples showed insulating behavior with a very high resistivity $(\rho)\left(\sim 10^{12} \Omega-\mathrm{cm}\right)$ at room temperature, independent of substrate and orientation.

To gain insight into the presence of cation ordering, we measured the magnetization $(M)$ versus temperature $(T)$ and applied magnetic field $(H)$. Figures $3 \mathrm{a}$ and $\mathrm{b}$ show the in-plane normalized field cooled $M(T)(H=200 \mathrm{Oe})$ for the (001) and (111)-oriented films, respectively. The $T_{C}$ values were determined as the minima of $\mathrm{d} M / \mathrm{d} T$ (see Table 1). The corresponding in-plane $M(H)$ loops at $5 \mathrm{~K}$ along the $[1 \overline{1} 0]$ direction are shown in Figures $3 \mathrm{c}$ and $\mathrm{d}$. To enhance the clarity of the $M(H)$ data, we subtracted the paramagnetic background that was only present for the films on $\mathrm{SrTiO}_{3}$. An overview of the main outcomes is shown in Table 1.

Taking a closer look at the $M(T)$ data, a well-defined magnetic transition was found $\sim 200 \mathrm{~K}$ for $\mathrm{La}_{2} \mathrm{CoMnO}_{6}$ on (111)-oriented substrates. On the (001)-oriented substrates, a minimum in the $\mathrm{d} M / \mathrm{d} T$ was also present, but at much lower temperatures, around $120 \mathrm{~K}$. Moreover, a clear tail is present in the $M(T)$ curve above $T_{\mathrm{C}}$, which is likely the result of a large proportion of $\mathrm{Co}-\mathrm{O}-\mathrm{Co}$ and $\mathrm{Mn}-\mathrm{O}-\mathrm{Mn}$ bonds leading to $\mathrm{AFM} /$ paramagnetic superexchange interaction. ${ }^{35,53,54}$ In addition, $M(H)$ shows a slight biloop behavior (Figure 3d) with a much lower $M_{S}$. This suggests the presence of disordered magnetic domains with a variance of magnetic coupling due to cation disordering.

The clear difference in $T_{\mathrm{C}}$ found between the (001) and the (111)-oriented films, $\sim 120 \mathrm{~K}$ and $\sim 200 \mathrm{~K}$, respectively, indicates that the substrate orientation strongly influences the magnetic properties of the film and likely the $B$-site cation arrangement. In previous studies, the high $T_{\mathrm{C}}$ was ascribed to $\mathrm{Co} / \mathrm{Mn}$ ordering, while the low $T_{\mathrm{C}}$ was believed to result from cation disorder, with the presence of $\mathrm{Mn}^{3+}$ and $\mathrm{Co}^{3+}$ and/or oxygen vacancies playing an important role. ${ }^{25,30,37}$ A reduction in $\mathrm{Co} / \mathrm{Mn}$ ordering in our (001)-oriented film is in full agreement with the hypothesis as the creation of two different $B$-site spaces would be absent for these films.

To understand the $M(T)$ behavior in more detail, we fitted the paramagnetic susceptibility $(\chi)$ of $\mathrm{La}_{2} \mathrm{CoMnO}_{6}$ on LSAT (111), the highest $T_{\mathrm{C}}$ sample, to a Curie-Weiss Law in the temperature range of $216-250 \mathrm{~K}$ (inset in Figure 3a, shown by the solid black line). A paramagnetic Curie temperature $(\theta)$ of $213 \pm 0.3 \mathrm{~K}$ was found.
Moreover, we used the expression $M \sim\left(T_{\mathrm{C}}-T\right)^{\beta}$, where $\beta$ is the critical exponent, to fit the $M(T)$ curves for comparison with simple mean field theory for spontaneous magnetization near $T_{\mathrm{C}}$ (inset Figure $3 \mathrm{a}$, blue solid line). $\beta$ and $T_{\mathrm{C}}$ were found to be $0.34 \pm 0.01$ and $211 \pm 1 \mathrm{~K}$, respectively. The values found for $T_{C}$ and $\theta$ are similar indicating ferromagnetic behavior of $\mathrm{La}_{2} \mathrm{CoMnO}_{6}$ on LSAT (111). The $T_{\mathrm{C}}$ is slightly lower than that observed in bulk $\mathrm{La}_{2} \mathrm{CoMnO}_{6}$. However, biaxial strain and a relatively low film thickness may reduce $T_{\mathrm{C}}$ as they can affect the magnetic ordering, the $B$-O bond length and/or $B-\mathrm{O}-B$ ' bond angle. ${ }^{55,56}$ The value found for $\beta$ was 0.34 which is close to a three dimensional isotropic Heisenberg ferromagnet. Similar behavior has been suggested for ordered $\mathrm{La}_{2} \mathrm{NiMnO}_{6}{ }^{35}$ The observation of high-quality ferromagnetic behavior indicates that the film has a very high degree of $\mathrm{Co} / \mathrm{Mn}$ ordering.

While $M(T)$ data show clear differences between (001)- and (111)-oriented films, the $M(H)$ loops show clear differences in $M_{s}$ and coercive field $\left(H_{\mathrm{c}}\right)$ between $\mathrm{La}_{2} \mathrm{CoMnO}_{6}$ on LSAT (111) and the other films (Figures $3 \mathrm{c}$ and d). The $\mathrm{La}_{2} \mathrm{CoMnO}_{6}$ films on (001) and (111) $\mathrm{SrTiO}_{3},(001)$ LSAT and (111) $\mathrm{LaAlO}_{3}$ showed a $M_{s}$ of $\sim 3.5-4 \mu_{\mathrm{B}} /$ f.u. with a $H_{c}$ of 9-13 kOe. These $M_{s}$ values are much lower than for ordered $\mathrm{La}_{2} \mathrm{CoMnO}_{6}$, suggesting the presence of $\mathrm{Co} / \mathrm{Mn}$ disorder. However, $M_{s}$ does not give any insight on the type of disorder. On the other hand, since the $T_{\mathrm{C}}$ ' values differ significantly between the (001) and (111)-oriented films, the $\mathrm{Co} / \mathrm{Mn}$ (dis)order is likely to be different. The presence of disorder is also in agreement with the enhancement in $H_{c}$ compared to (partially) ordered $\mathrm{La}_{2} \mathrm{CoMnO}_{6}$ reported in previous studies (5-7 kOe). ${ }^{36,39}$ The enhancement in $H_{\mathrm{c}}$ can be explained by the presence of anti-phase domains.

$\mathrm{La}_{2} \mathrm{CoMnO}_{6}$ on LSAT (111) showed a much higher $M_{s}$ compared to all other films studied, $M_{s} \sim 5.8 \mu_{\mathrm{B}} /$ f.u., which is close to the theoretical maximum value of $6 \mu_{\mathrm{B}} /$ f.u. for fully ordered $\mathrm{La}_{2} \mathrm{CoMnO}_{6}$, as well as a relatively low $H_{\mathrm{c}} \sim 6 \mathrm{kOe}$, indicating a high degree of $\mathrm{Co} / \mathrm{Mn}$ order. In comparison to previously reported ordered $\mathrm{La}_{2} \mathrm{CoMnO}_{6}$ films, our films on LSAT (111) showed a clear saturation in magnetization above $25 \mathrm{kOe}$. In previous studies, saturation was not even observed up to $40 \mathrm{kOe}$, probably due to the presence of AFM interactions. ${ }^{25,36,39}$ As the absence in saturation was not taken into account for determining the $M_{\varsigma}$ in those studies, the actual $M_{\varsigma}$ is likely to be lower than reported, and therefore also the $\mathrm{Co} / \mathrm{Mn}$ ordering. In addition, from the $M(H)$ loop, there is no evidence for the presence of other magnetic domains resulting from $B$-site cation disordering or the presence of antisite defects. ${ }^{54}$ As our films on LSAT (111) show a clear saturation in the magnetization with a $M_{s}$ of $\sim 5.8 \mu_{\mathrm{B}} /$ f.u. in combination with a high $T_{\mathrm{C}}$ and a well-defined $M(H)$ loop, a high degree of $\mathrm{Co} / \mathrm{Mn}$ ordering has been achieved in these films.

To get a better understanding of the $\mathrm{La}_{2} \mathrm{CoMnO}_{6}$ structure, chemical ordering and the resulting magnetic behavior, we compared the films grown on $\mathrm{SrTiO}_{3}$ (111) and LSAT (111) using analytical STEM. Both films showed very good epitaxy and a well-defined interface with the substrate as shown in the high-angle annular dark field image $\mathrm{La}_{2} \mathrm{CoMnO}_{6}$ on LSAT and the low angle annular dark field image for $\mathrm{La}_{2} \mathrm{CoMnO}_{6}$ on $\mathrm{SrTiO}_{3}$ (Figures 4a and e), which is in agreement with our XRD results. Moreover, the characteristic partial Al-Ta ordering in the LSAT substrate is clearly visible. ${ }^{57}$ Please note that these images have an exceptionally high signal to noise level without global or local distortions due to the acquisition of a sequence of short exposure scans followed by the application of rigid and non-rigid registration procedures, as described in detail in the 'Experimental section'. Therefore these data are suited to use for quantitative analysis of atomic positions. 

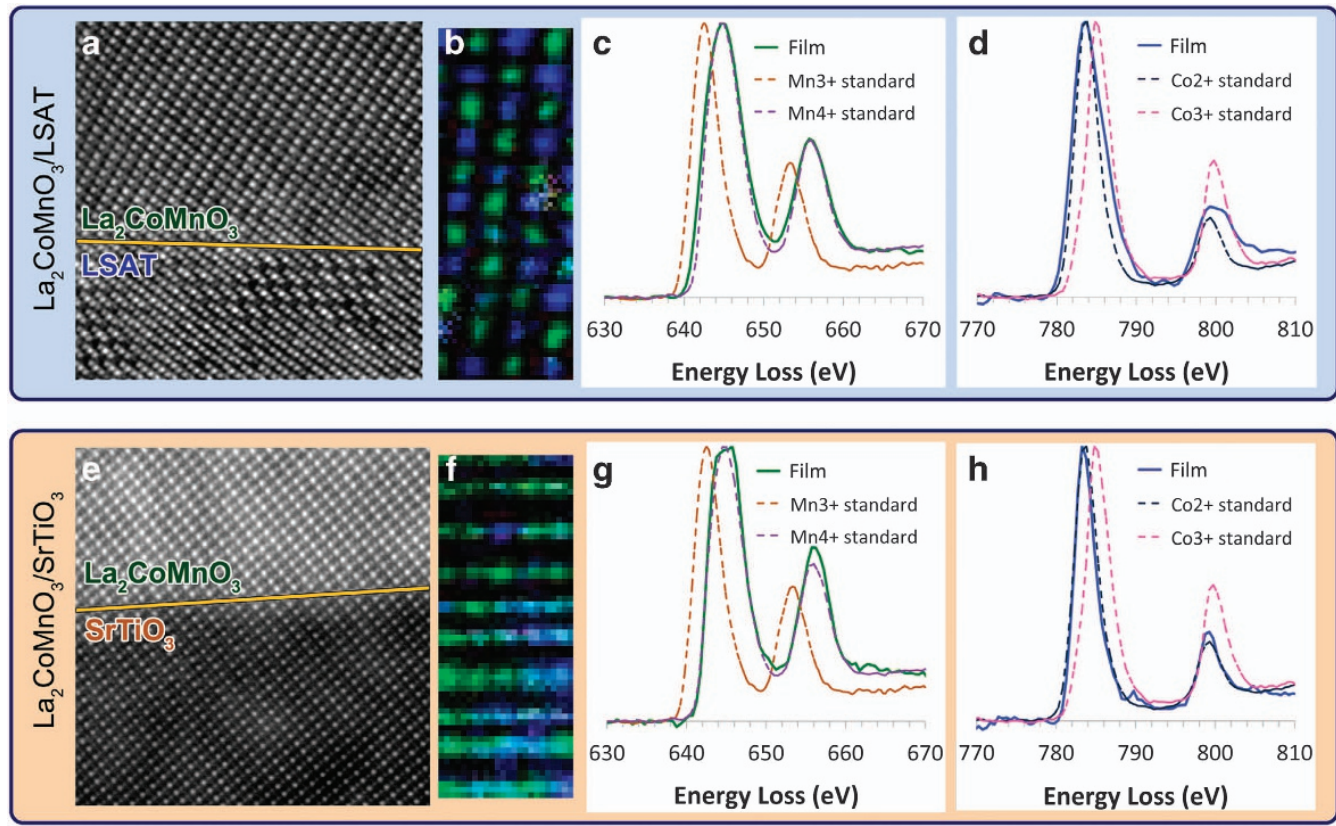

Figure 4 STEM of the structure and chemistry of $\mathrm{La}_{2} \mathrm{CoMnO}_{6}$ films on (111)-oriented LSAT and (111)-oriented SrTiO $\mathrm{S}_{3}$ (a) high-angle annular dark field STEM image of $10 \mathrm{~nm}^{2}$ of the substrate-film interface for the $\mathrm{La}_{2} \mathrm{CoMnO}_{6}$ film on LSAT, with the approximate interface position delineated, the partial Al-Ta ordering in the LSAT is easily seen; (b) superimposed EELS maps of $\mathrm{Mn}$ (green) and $\mathrm{Co}$ (blue) showing a high degree of ordering for the La $\mathrm{CoMnO}_{6}$ film on LSAT; (c) detailed EEL spectrum around the $\mathrm{Mn}-\mathrm{L}_{2,3}$ edge for the bulk of the $\mathrm{La}_{2} \mathrm{CoMnO}_{6}$ film on LSAT compared to standards; (d) detailed EEL spectrum around the $\mathrm{Co}_{-} \mathrm{L}_{2,3}$ edge from the same area as (c) compared to standards; (e) low-angle annular dark field-STEM image of $10 \mathrm{~nm}^{2}$ of $\mathrm{La}_{2} \mathrm{CoMnO}_{6}$ on $\mathrm{SrTiO}_{3}$, with the approximate interface position delineated; (f) superimposed $\mathrm{Mn}$ (green) and Co (blue) EELS maps of an area within the centre of the film on $\mathrm{SrTiO}_{3}$ showing some columns richer in one element, but no clear evidence of long-range Co-Mn ordering; (g) detailed EEL spectrum around the Mn- $\mathrm{L}_{2,3}$ edge for the bulk of the $\mathrm{La}_{2} \mathrm{CoMnO}_{6}$ film on $\mathrm{SrTiO}_{3}$ compared to standards; (h) detailed $\mathrm{EEL}$ spectrum around the $\mathrm{Co}-\mathrm{L}_{2,3}$ edge from the same area as $(\mathbf{g}$ ) compared to standards.

To determine the type of ordering, spatially resolved electron energy loss spectroscopy spectrum imaging was undertaken on both samples. A clear difference in $B$-site ordering between the two films was present. For the film grown on (111) LSAT, the superimposed electron energy loss spectroscopy spectrum imaging maps of $\mathrm{Mn}$ and Co showed a clear rock salt ordering pattern, indicating that the bulk of the film has a high degree of long-range rock salt ordering (Figure $4 \mathrm{~b}$ ). Furthermore, the high degree of cation ordering is in agreement with the extracted electron energy loss spectroscopy (EELS) spectra from this region, which match well to standard spectra for $\mathrm{Co}^{2+}$ and $\mathrm{Mn}^{4+}$ (Figures $4 \mathrm{c}$ and $\mathrm{d}$ ). This is consistent with previous measurements that suggest that atomic ordering is associated with strong charge ordering. ${ }^{30}$ For the film grown on (111) $\mathrm{SrTiO}_{3}$, the ordering is less clearly visible (Figure 4f). However, some columns still clearly have more Co or $\mathrm{Mn}$, and the EEL spectra still match well to standard spectra for $\mathrm{Co}^{2+}$ and $\mathrm{Mn}^{4+}$ (Figures $4 \mathrm{~g}$ and $\mathrm{h}$ ), suggesting that a high degree of local ordering of $\mathrm{Mn}$ and Co is still present in the film on $\mathrm{SrTiO}_{3}$ (111), even if cation ordering does not persist over length scales longer than several nanometers, suggesting a nanoscale ordering domain structure. Multiple nanosized anti-phase domains have been inferred in other DPs as well. ${ }^{58}$ Moreover, a short-range ordered structure would also clarify the significantly reduced $M_{s}$ as a result of the AFM $\mathrm{Co}^{2+}-\mathrm{Co}^{2+}$ and $\mathrm{Mn}^{4+}-\mathrm{Mn}^{4+}$ interactions at the anti-phase domains boundaries. However, since the degree of local cation order remained very high, only a minor reduction of $T_{C}$ is present. ${ }^{59}$

Our hypothesis states that the presence of anti-phase octahedral rotations would be required to enhance cation ordering. For both films, the oxygen maps in the bulk of the film show a clear zig-zag pattern along the $[1 \overline{1} 0]_{\mathrm{pc}}$ direction consistent with the expected octahedral tilt pattern, as shown in Figure $5 \mathrm{a}$ for $\mathrm{La}_{2} \mathrm{CoMnO}_{6}$ on LSAT. This pattern is also seen in ABF STEM images of the interface for $\mathrm{La}_{2} \mathrm{CoMnO}_{6}$ on $\mathrm{SrTiO}_{3}$ (111) (Figure 5b). Quantification of the ABF STEM images of the $\mathrm{La}_{2} \mathrm{CoMnO}_{6}$ film on $\mathrm{SrTiO}_{3}$ show that anti-phase rotation along the $[100]_{\mathrm{pc}}$ and $[010]_{\mathrm{pc}}$ immediately starts at the interface and increases in strength over a few $(111)_{\mathrm{pc}}$ planes until it reaches a plateau with a total tilt angle of $\pm 8^{\circ}$ (Figure $5 \mathrm{c}$ ). See Supplementary Information for simulations that confirm this intuitive interpretation of the ABF images. (See supporting information for details regarding the interpretation of the ABF images.) The presence of octahedral tilting in both films is consistent with the presence of cation ordering.

To understand the absence of long-range ordering in $\mathrm{La}_{2} \mathrm{CoMnO}_{6}$ on $\mathrm{SrTiO}_{3}$ (111), we also investigated the pseudocubic layer spacing by doing quantitative measurements of the spacing between the $A$-site layers $(d)$ along the out-of-plane direction, as the $d$-spacing is linked to the size and shape of the $B$-site cage.

In the $\mathrm{SrTiO}_{3}$ substrate, $A$-site layers are spaced with the expected $d$-spacing of $\sim 2.25 \AA$. In the $\mathrm{La}_{2} \mathrm{CoMnO}_{6}$ film on $\mathrm{SrTiO}_{3}$, these spacings develop a pattern of alternating short and long $d$-spacings, as shown in Figure 5e, where the alternating spacing increases over about $20(111)_{\mathrm{pc}}$ layers to values of around 2.32 and $2.18 \AA$. As this modulation of $A$-site $d$-spacings correlates very well with the trend in tilting pattern, it is clear that $d$-spacing and octahedral tilting are coupled. This is in agreement with the results for bulk ordered $\mathrm{La}_{2} \mathrm{CoMnO}_{6}$, where a modulation of alternate spacings of (101) monoclinic planes of 2.37 and $2.14 \AA$ is present. ${ }^{29}$ Comparing the $d$-spacings with the bulk values, it is clear that the observed 

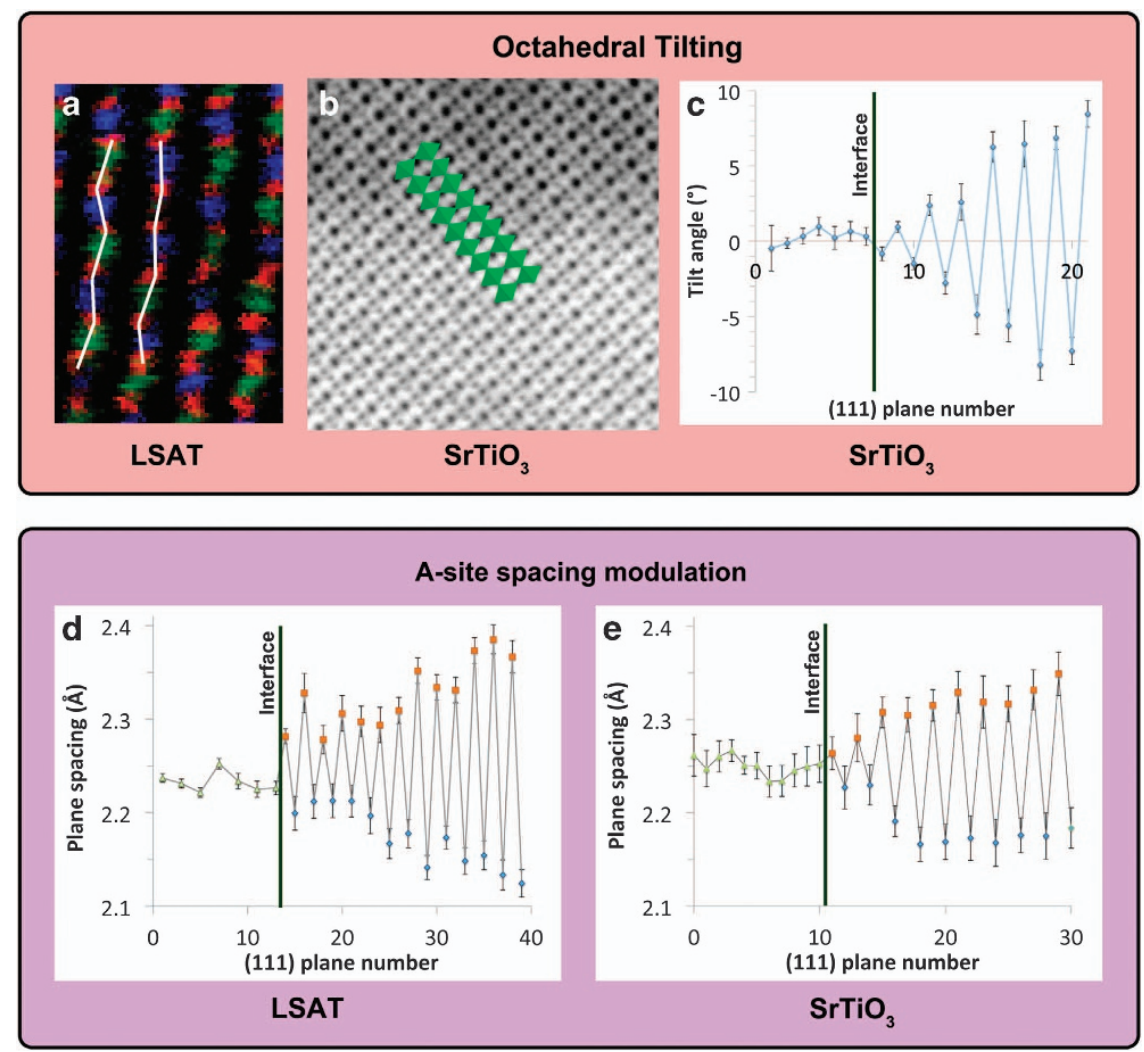

Figure 5 (a) Superimposed EELS maps of oxygen (red), $\mathrm{Mn}$ (green) and $\mathrm{Co}$ (blue) of $\mathrm{La}_{2} \mathrm{CoMnO}_{6}$ on LSAT from the same area as Figure $4 \mathrm{~b}$ showing a clear zig-zag pattern of the oxygen positions, meaning strong correlated octahedral tilting; (b) ABF image $\left(5 \mathrm{~nm}^{2}\right)$ of $\mathrm{La}_{2} \mathrm{CoMnO}_{6}$ on $\mathrm{SrTiO}_{3}$ at the interface showing a clear zig-zag of the oxygen positions, which is consistent with octahedral tilting; (c) plot of tilt angle for the octahedral tilting going from the $\mathrm{SrTiO}_{3}$ substrate into $\mathrm{La}_{2} \mathrm{CoMnO}_{6}$, the dotted line is a guide-to-the-eye; (d) plot of $(111)_{\mathrm{pc}}$ spacings between $A$-site layers going from the LSAT substrate into the $\mathrm{La}_{2} \mathrm{CoMnO}_{6}$ film, the dotted line is a guide-to-the-eye; and (e) plot of $(111)_{\mathrm{pc}}$ spacings between $A$-site layers going from the $\mathrm{SrTiO}_{3}$ substrate into the $\mathrm{La}_{2} \mathrm{CoMnO}_{6}$ film, the dotted line is a guide-to-the-eye.

modulation in the film on $\mathrm{SrTiO}_{3}$ (111) is approaching the bulk values, but did not reach the full modulation after $20(111)_{\mathrm{pc}}$ layers.

A similar effect in $d$-spacings is seen for $\mathrm{La}_{2} \mathrm{CoMnO}_{6}$ on LSAT (111) (Figure 5d). In the area analyzed, significant $\mathrm{Al}-\mathrm{Ta}$ ordering was present in the LSAT substrate, resulting in a period doubling of $(111)_{\mathrm{pc}}$ planes in the substrate. Therefore, for ease of comparison, the spacing of $(111)_{\mathrm{pc}}$ planes in LSAT is calculated by halving the actual plane spacing. This averaged spacing is very uniform in LSAT with a constant value of $2.23 \AA$. In $\mathrm{La}_{2} \mathrm{CoMnO}_{6}$, a bimodal distribution of $(111)_{\mathrm{pc}}$ layer spacings builds up immediately at the interface, similar as for the film on $\mathrm{SrTiO}_{3}$. However, the bimodality of layer spacings for $\mathrm{La}_{2} \mathrm{CoMnO}_{6}$ on LSAT (111) is much stronger approaching bulk values of 2.14 and $2.37 \AA$ at $\sim 20$ layers away from the interface.

Our data clearly show that the $d$-spacing modulation in $\mathrm{La}_{2} \mathrm{CoMnO}_{6}$ on LSAT is larger than on $\mathrm{SrTiO}_{3}$. This is in agreement with the higher degree of ordering observed on LSAT. The small compressive strain sets up a pattern of co-operative octahedral tilting and $A$-site layer spacing modulation resulting in a large difference in the size of the $B$-site cages within the octahedra in each alternating layer along the growth direction. The pronounced difference in $B$-site cages then has the simple consequence that the smaller $\mathrm{Mn}^{4+}$ ions are attracted to the smaller octahedra and the larger $\mathrm{Co}^{2+}$ ions to the larger ones, giving the observed ordering pattern and resulting properties.

The minor tensile strain of $\mathrm{SrTiO}_{3}$ appears to slightly suppress the out-of-plane $d$-spacing modulation. This suppression may be due to the change in orientation of the monoclinic $\mathrm{La}_{2} \mathrm{CoMnO}_{6}$ unit cell $\left(a_{\text {monoclinic }}=0.553 \mathrm{~nm}, b_{\text {monoclinic }}=0.549 \mathrm{~nm}\right)$ with respect to the out-of-plane direction when moving from compressive (on LSAT) to tensile strain (on $\mathrm{SrTiO}_{3}$ ). The growth of $\mathrm{La}_{2} \mathrm{CoMnO}_{6}$ on (111) could proceed with a number of different orientations of the monoclinic unit cell with respect to the substrate. Firstly, any of the directions in the monoclinic cell equivalent to $[111]_{\text {cubic }}$ could appear as the out-of-plane direction. By analyzing the lattice mismatch between the three directions equivalent to $\langle 110\rangle_{\text {cubic }}$ directions in the interface plane, it is possible to find the orientation that gives the lowest mismatch and therefore lowest strain (the same principle was used previously for predicting domain assemblages, for example by MacLaren et al.). ${ }^{60}$ It is found that of the four distinct $\langle 111\rangle_{\text {cubic }}$

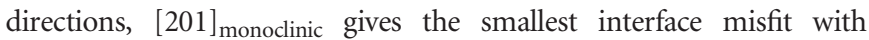
$-0.61 \%,-0.61 \%$ and $-0.36 \%$ misfit along the three in-plane $<110\rangle_{\text {cubic }}$ directions for the film grown on LSAT. There are then three rotational variants of this orientation, with the $[010]_{\text {monoclinic }}$ direction with the low $-0.36 \%$ misfit along any of the three crystallographically equivalent $\langle 110\rangle_{\text {cubic }}$ in the substrate at the interface. Thus, a domain structure of three crystallographically equivalent variants would be expected with the $[010]_{\text {monoclinic }}$ direction in-plane in all cases for films grown on LSAT. For films grown on $\mathrm{SrTiO}_{3}$, the misfit is lower and the strain is lowest for [021] and $[02 \overline{1}]$ orientations of the film normal $(0.27,-0.14$ and $0.34 \%$ for the three in-plane directions in both cases). The lack of a strong constraint from $\mathrm{SrTiO}_{3}$ would therefore allow the nucleation of a 

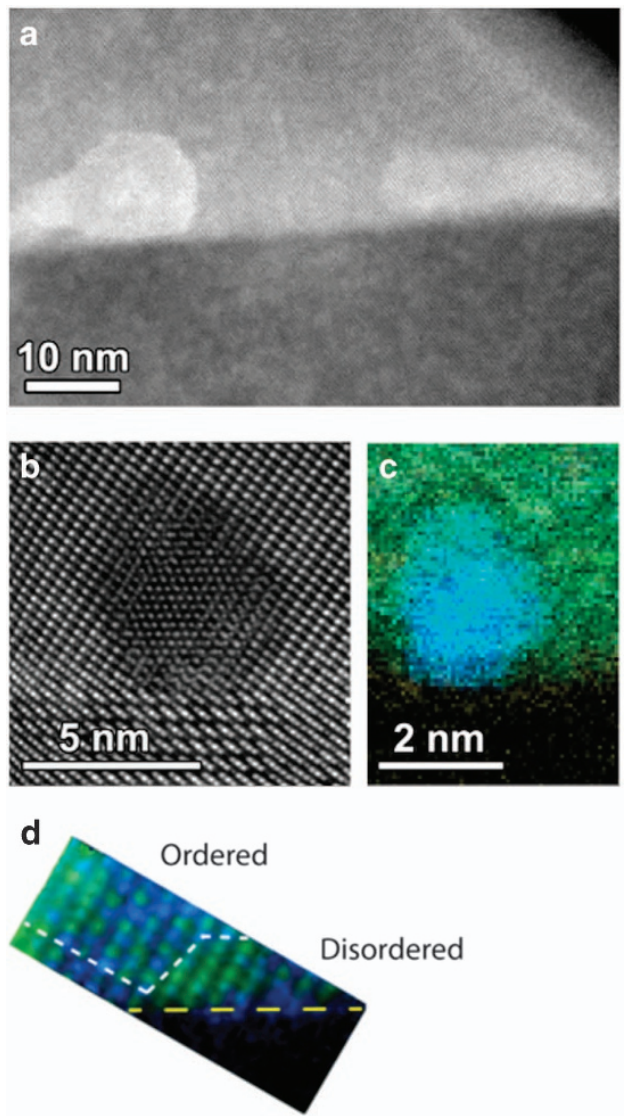

Figure 6 STEM imaging and electron energy loss spectroscopy spectrum imaging of non-stoichiometry at the substrate-film interface: (a) high-angle annular dark field-STEM image of Co-rich particles at the interface of $\mathrm{La}_{2} \mathrm{CoMnO}_{6}$ on $\mathrm{SrTiO}_{3}$; (b) atomic resolution high-angle annular dark-fieldSTEM image of the atomic structure of a particle in the $\mathrm{La}_{2} \mathrm{CoMnO}_{6}$ film on LSAT; (c) superimposed Co-Mn EELS map of the particle in $\mathbf{b}$, where Co is blue and $\mathrm{Mn}$ is green; (d) superimposed Co-Mn EELS map, where Co is blue and $\mathrm{Mn}$ is green, of an interface area on LSAT that was free of Co-rich particles. The yellow dotted line indicates the interface between LSAT and $\mathrm{La}_{2} \mathrm{CoMnO}_{6}$.

more complex domain structure, which may also reduce the driving force for strong elemental and magnetic ordering. This could also explain the difference in $d$-spacing modulation between the films on LSAT (111) and $\mathrm{SrTiO}_{3}$ (111) (Figures 5d and e).

Growth of $\mathrm{La}_{2} \mathrm{CoMnO}_{6}$ on both LSAT and $\mathrm{SrTiO}_{3}$ substrates produced a very small concentration of precipitates at the substrate/ film interface (Figure 6). The precipitates on $\mathrm{SrTiO}_{3}$ are, on average, slightly larger $(\sim 10 \mathrm{~nm}$, Figure $6 \mathrm{a})$ compared to those on LSAT $(\sim 2-5 \mathrm{~nm}$, Figure $6 \mathrm{~b})$. Nevertheless, the precipitates rarely grow large or persist for any significant thickness of the film. On the basis of a combination of EELS and atomic resolution high-angle annular dark-field STEM imaging, the precipitates are most likely to be rock salt structured $\mathrm{CoO}$, with the $[111]_{\mathrm{CoO}}$ directed out-of-plane (Figures $6 \mathrm{~b}$ and $\mathrm{c}$ ). As this phase was not observed by XRD, the volume fraction is very small. The particles being $\mathrm{CoO}$ agrees with the observed impurity phase seen in XRD by Egoavil et al. ${ }^{39}$. The presence of $\mathrm{CoO}$ is consistent with the fact that the first few layers of $\mathrm{La}_{2} \mathrm{CoMnO}_{6}$ were Mn-rich and Co-poor with $\mathrm{Mn}$ :Co ratios up to 70\%:30\% as determined by EELS quantification. However, since $\mathrm{La}_{2} \mathrm{Co}_{1-x} \mathrm{Mn}_{1+x} \mathrm{O}_{6}$ also has a ferromagnetic $T_{\mathrm{C}} \sim 210 \mathrm{~K}$, no effect on the $M(T)$ has been observed. ${ }^{61,62}$ The deviation in stoichiometry also means that the ideal rock salt ordering is disturbed in those first few layers near the interface (Figure 6d), which is consistent with previous studies. ${ }^{39}$ The formation of $\mathrm{CoO}$ and $\mathrm{La}_{2} \mathrm{Mn}_{1+x} \mathrm{Co}_{1-x} \mathrm{O}_{6}$ can be explained by the fact that the in-plane strained lattice sites are too small for $\mathrm{Co}^{2+}$ in the first few layers, until the octahedral tilting and $A$-site spacing modulation has built up to the bulk-like values, as shown in Figures $5 \mathrm{~d}$ and e. This also explains why the ideal ordered $\mathrm{La}_{2} \mathrm{CoMnO}_{6}$ structure forms readily, as shown in Figure $6 \mathrm{~d}$. Furthermore, the ordered $\mathrm{La}_{2} \mathrm{CoMnO}_{6}$ grows over the top of the $\mathrm{CoO}$ defects leaving the bulk of the film as the ideal rock salt ordered DP structure.

With a good understanding of the $\mathrm{Co} / \mathrm{Mn}$ ordering of $\mathrm{La}_{2} \mathrm{CoMnO}_{6}$ on $\mathrm{SrTiO}_{3}$ (111) and LSAT (111), we are now able to infer the type of (dis)order in the films on $\mathrm{LaAlO}_{3}(111), \mathrm{SrTiO}_{3}$ (001) and LSAT (001). First of all, since the magnetic behavior of $\mathrm{La}_{2} \mathrm{CoMnO}_{6}$ on $\mathrm{LaAlO}_{3}$ (111) and $\mathrm{SrTiO}_{3}$ (111) is comparable, a similar type of $\mathrm{Co}-\mathrm{Mn}$ ordering can be expected, that is, short-range ordering with nanometer sized anti-phase domains. The absence of long-range cation ordering on $\mathrm{LaAlO}_{3}$ can be explained by the absence of induced in-plane strain as a result of a too large in-plane mismatch. As a consequence, the strain-induced effect required to promote the formation of two different $B$-site cages was absent. However, one may still expect the formation of the out-of-plane $A$-site $d$-spacing modulation as for the film on $\mathrm{SrTiO}_{3}$, which arises as a natural result of the octahedral tilting due to growing along the [111] direction.

A drastic change in the magnetic behavior of $\mathrm{La}_{2} \mathrm{CoMnO}_{6}$ was found by changing the orientation of the substrate from (111) to (001). For the (001)-oriented films, both a low $T_{\mathrm{C}}$ and a low $M_{s}$ with large $H_{\mathrm{C}}$ were found, as well as a clear tail in the $M(T)$ was present indicating the presence of AFM interactions. This strongly suggests a highly disordered or randomly ordered cation structure on the $B$-site. In addition, the reduced $T_{\mathrm{C}}$ may indicate the presence of oxygen vacancies. ${ }^{30}$ Note that the formation of oxygen vacancies can depend on the film growth direction, even though the film growth conditions are the same. ${ }^{63}$ Recently, calculations have shown that oxygen vacancies are more likely to form in $\mathrm{La}_{2} \mathrm{CoMnO}_{6}$ when Co and $\mathrm{Mn}$ are mainly in the $3+$ oxidation state, ${ }^{63}$ which would be expected for a random distribution of $\mathrm{Co}$ and $\mathrm{Mn}$ ions on the $B$-sites. Moreover, the presence of oxygen vacancies would also stimulate an increase in the formation of $\mathrm{Mn}^{3+}$, that is, increasing the degree of $\mathrm{Co} / \mathrm{Mn}$ disorder. ${ }^{30}$ Therefore, the films grown on the (001) substrates are likely to be highly disordered and oxygen vacancy rich.

Finally, with the understanding of the differences in nature of cation (dis)order for the various samples, we now focus on why these different types of ordering occur. Therefore, we return to the hypothesis that states that two different octahedra, that is, two different $B$-site cages, can be formed when growing distorted perovskites on (111)-oriented substrates and thereby driving cation ordering. The hypothesis is in full agreement with our results as a significant increase in $\mathrm{Co} / \mathrm{Mn}$ ordering in films on (111)-oriented substrates was observed compared to the films on (001)-oriented substrates. For the (001)-oriented films cation ordering was not stimulated by the substrate orientation. Since in this study, cation ordering was not stimulated by the growth conditions, no cation ordering was detected in the (001)-oriented $\mathrm{La}_{2} \mathrm{CoMnO}_{6}$ films.

\section{CONCLUSION}

To conclude, spontaneous $B$-site rock salt cation ordering can be stimulated in DP thin films by coherent growth along the [111] direction. This is because the combination of internal octahedral rotations of the DP and the in-plane strain from the (111)-oriented 
substrate induces the formation of two differently sized $B$-site spaces in a rock salt arrangement. A clear increase in $\mathrm{Co} / \mathrm{Mn}$ rock salt ordering was achieved by growing on (111)-oriented substrates, including on $\mathrm{SrTiO}_{3}$ and LSAT. In addition, we have shown that cation ordering is strongly enhanced in $\mathrm{La}_{2} \mathrm{CoMnO}_{6}$ under a minor in-plane compressive strain when grown on a suitable (111)-oriented substrate, resulting in long-range $\mathrm{Co} / \mathrm{Mn}$ ordered films with a $M_{s}$ of $5.8 \mu_{\mathrm{B}} /$ f.u. Since the growth on (111)-oriented substrates can stimulate cation ordering and the driving force is insensitive to growth conditions, our work opens up the possibility of the realization of a whole range of new, high-quality functional DP thin films for a range of applications such as magneto-optic, spin-filter and magnetoelectric devices.

\section{CONFLICT OF INTEREST}

The authors declare no conflict of interest.

\section{ACKNOWLEDGEMENTS}

This work was supported by the European Research Council (ERC) (Advanced Investigator grant ERC-2009-AdG-247276-NOVOX), the EPSRC (Equipment Account Grant EP/K035282/1) and the Isaac Newton Trust (Minute 13.38(k)). LJ thank the support of the European Union Seventh Framework Programme under Grant Agreement 312483-ESTEEM2 (Integrated Infrastructure Initiative-I3). SuperSTEM is the UK National Facility for Aberration Corrected STEM funded by EPSRC.

Author contributions: JEK and JLMD designed the project. JEK and EDJ have grown the films. JEK and EDJ have done the XRD measurements; JEK, EMC and EDJ have done the magnetic measurements. DK and DHM acquired the STEM images and spectral data, with technical input and oversight from QMR. Additional STEM images and spectral data were acquired at Glasgow by IM. LJ performed the non-rigid registration of the STEM data sets. STEM data analysis was performed by TMY, BS, BAM, DK and DHM under the leadership of IM. JB performed STEM image simulations to validate the conclusions made in the STEM data analysis. All authors contributed to the discussions and writing of the article.

1 Liu, M., Ma, C., Collins, G., Liu, J., Chen, C., Alemayehu, A. D., Subramanyam, G., Ding, Y., Chen, J., Dai, C., Lin, Y. \& Cole, M. W. Ferroelectric $\mathrm{BaTiO}_{3} / \mathrm{SrTiO}_{3}$ multilayered thin films for room-temperature tunable microwave elements. Nanoscale Res. Lett. 8, 338 (2013).

2 Nechache, R., Harnagea, C., Li, S., Cardenas, L., Huang, W., Chakrabartty, J. \& Rosei, F. Bandgap tuning of multiferroic oxide solar cells. Nat. Photon. 9, 61-67 (2015).

3 Das, H., De-Raychaudhury, M. \& Saha, T. Moderate to large magneto-optical signals in high $T_{C}$ double perovskites. Appl. Phys. Lett. 92, 201912 (2008)

4 Hashisaka, M., Kan, D., Masuno, A., Terashima, T., Takano, M. \& Mibu, K. Spinfiltering effect of ferromagnetic semiconductor $\mathrm{La}_{2} \mathrm{NiMnO}_{6}$. J. Magn. Magn. Mater. 310, 1975-1977 (2007).

5 Bousquet, E., Dawber, M., Stucki, N., Lichtensteiger, C., Hermet, P., Gariglio, S., Triscone, J.-M. \& Ghosez, P. Improper ferroelectricity in perovskite oxide artificial superlattices. Nature 452, 732-736 (2008).

6 Tabata, H., Tanaka, H. \& Kawai, T. Formation of artificial $\mathrm{BaTiO}_{3} / \mathrm{SrTiO}_{3}$ superlattices using pulsed laser deposition and their dielectric properties. Appl. Phys. Lett. 65 1970 (1994).

7 Blasse, G. Ferromagnetic interactions in non-metallic perovskites. J. Phys. Chem. Solids 26, 1969-1971 (1965).

8 Azuma, M., Takata, K., Saito, T., Ishiwata, S., Shimakawa, Y. \& Takano, M. Designed ferromagnetic, ferroelectric $\mathrm{Bi}_{2} \mathrm{NiMnO}_{6}$. J. Am. Chem. Soc. 127, 8889-8892 (2005).

9 Kobayashi, K.-I., Kimura, T., Sawada, H., Terakura, K. \& Tokura, Y. Room-temperature magnetoresistance in an oxide material with an ordered double-perovskite structure. Nature 395, 677-680 (1998).

10 Rondinelli, J. M. \& Fennie, C. J. Octahedral rotation-induced ferroelectricity in cation ordered perovskites. Adv. Mater. 24, 1961-1968 (2012).

11 Berger, R. F. \& Neaton, J. B. Computational design of low-band-gap double perovskites. Phys. Rev. B 86, 165211 (2012).

12 Liu, Y. P., Chen, S. H., Tung, J. C. \& Wang, Y. K. Investigation of possible half-metal material on double perovskites $\mathrm{Sr}_{2} \mathrm{BBO}_{6}$ ( $B, B=3 d$ transition metal) using first-principle calculations. Solid State Commun. 152, 968-973 (2012).

13 Pickett, W. E. Spin-density-functional-based search for half-metallic antiferromagnets. Phys. Rev. B 57, 10613 (1998).
14 Galasso, F., Katz, L. \& Ward, R. Substitution in the octahedrally coördinated cation positions in compounds of the perovskite type. J. Am. Chem. Soc. B 81, 820-823 (1959).

15 Anderson, M. T., Greenwood, K. B., Taylor, G. A. \& Poeppelmeier, K. R. B-cation arrangements in double perovskites. Prog. Solid State Chem. 22, 197-233 (1993).

16 Hauser, A., Robert, J., Williams, E. A., Ricciardo, R. A., Genc, A., Dixit, M., Lucy, J. M., Woodward, P. M., Fraser, H. L. \& Yang, F. Unlocking the potential of half-metallic $\mathrm{Sr}_{2} \mathrm{FeMoO}_{6}$ films through controlled stoichiometry and double-perovskite ordering. Phys. Rev. B 83, 014407 (2011).

17 Galceran, R., Frontera, C., Balcells, L., Cisneros-Fernández, J., López-Mir, L., Roqueta, J., Santiso, J., Bagués, N., Bozzo, B., Pomar, A., Sandiumenge, F. \& Martínez, B. Engineering the microstructure and magnetism of $\mathrm{La}_{2} \mathrm{CoMnO}_{6-\delta}$ thin films by tailoring oxygen stoichiometry. Appl. Phys. Lett. 105, 242401 (2014).

18 Chakraverty, S., Ohtomo, A., Okuyama, D., Saito, M., Okude, M., Kumai, R., Arima, T., Tokura, Y., Tsukimoto, S., Ikuhara, Y. \& Kawasaki, M. Ferrimagnetism and spontaneous ordering of transition metals in double perovskite $\mathrm{La}_{2} \mathrm{CrFeO}_{6}$ films. Phys. Rev. B 84, 064436 (2011).

19 Chakraverty, S., Yoshimatsu, K., Kozuka, Y., Kumigashira, H., Oshima, M., Makino, T., Ohtomo, A. \& Kawasaki, M. Magnetic and electronic properties of ordered doubleperovskite $\mathrm{La}_{2} \mathrm{VMnO}_{6}$ thin films. Phys. Rev. B 84, 132411 (2011).

20 Yoshimatsu, K., Nogami, K., Watarai, K., Horiba, K., Kumigashira, H., Sakata, O., Oshima, T. \& Ohtomo, A. Synthesis and magnetic properties of double-perovskite oxide $\mathrm{La}_{2} \mathrm{MnFeO}_{6}$ thin films. Phys. Rev. B 91, 054421 (2015).

21 Nogami, K., Yoshimatsu, K., Mashiko, H., Sakai, E., Kumigashira, H., Sakata, O., Oshima, T. \& Ohtomo, A. Epitaxial synthesis and electronic properties of doubleperovskite $\mathrm{Sr}_{2} \mathrm{TiRuO}_{6}$ films. Appl. Phys. Express 6, 105502 (2013).

22 Chakraverty, S., Yu, X. Z., Kawasaki, M., Tokura, Y. \& Hwang, H. Y. Spontaneous B-site order and metallic ferrimagnetism in $\mathrm{LaSrVMoO}_{6}$ grown by pulsed laser deposition. Appl. Phys. Lett. 102, 222406 (2013).

23 Palkar, V. R. \& Malik, A. K. Observation of magnetoelectric behavior at room temperature in $\mathrm{Pb}\left(\mathrm{FexTi}_{1-x}\right) \mathrm{O}_{3}$. Solid State Commun. 134, 783-786 (2005).

24 Manako, T., Izumi, M., Konishi, Y. \& Kobayashi, K.-I. Epitaxial thin films of ordered double perovskite $\mathrm{Sr}_{2} \mathrm{FeMoO}_{6}$. Appl. Phys. Lett. 74, 2215 (1999).

25 Guo, H. Z., Gupta, A., Calvarese, T. G. \& Subramanian, M. A. Structural and magnetic properties of epitaxial thin films of the ordered double perovskite $\mathrm{La}_{2} \mathrm{CoMnO}_{6}$. Appl. Phys. Lett. 89, 262503 (2006).

26 Glazer, A. M. The classification of tilted octahedra in perovskites. Acta Crystallogr. B 28, 3384-3392 (1972).

27 King, G. \& Woodward, P. M. Cation ordering in perovskites. J. Mater. Chem. 20, 5785-5796 (2010).

28 Vasala, S. \& Karpinnen, M. $A_{2} B^{\prime} B^{\prime \prime} O_{6}$ perovskites: a review. Prog. Solid State Chem. $\mathbf{4 3 ,}$ 1-36 (2015).

29 Bull, C. L., Gleeson, D. \& Knight, K. S. Determination of B-site ordering and structural transformationsin the mixed transition metal perovskites $\mathrm{La}_{2} \mathrm{CoMnO}_{6}$ andLa2 $\mathrm{NiMnO}_{6}$. J. Phys. Condens. Matter 15, 4927-4936 (2003).

30 Dass, R. I. \& Goodenough, J. B. Multiple magnetic phases of $\mathrm{La}_{2} \mathrm{CoMnO}_{6-\delta}(0<\sim \delta$ $<\sim 0.05)$. Phys. Rev. B 67, 014401 (2003).

31 Singh, M. P., Charpentier, S., Truong, K. D. \& Fournier, P. Evidence of bidomain structure in double-perovskite $\mathrm{La}_{2} \mathrm{CoMnO}_{6}$ thin films. Appl. Phys. Lett. 90, 211915 (2007).

32 Sayed, F. N. Role of annealing atmosphere on structure, dielectric and magnetic properties of $\mathrm{La}_{2} \mathrm{CoMnO}_{6}$ and $\mathrm{La}_{2} \mathrm{MgMnO}_{6}$. Z. Anorg. Allg. Chem. 640, 1907-1921 (2014).

33 Kyômen, T., Yamazaki, R. \& Itoh, M. Correlation between magnetic properties and Mn/ $\mathrm{Co}$ atomic order in $\mathrm{LaMn}_{0.5} \mathrm{CO}_{0.5} \mathrm{O}_{3+\delta}$. 2. magnetic and calorimetric properties. Chem. Mater. 16, 179-184 (2004).

34 Kyômen, T., Yamazaki, R. \& Itoh, M. Correlation between magnetic properties and Mn $\mathrm{Co}$ atomic order in $\mathrm{LaMn}_{0.5} \mathrm{Co}_{0.5} \mathrm{O}_{3+\delta}$ : I. second-order nature in $\mathrm{Mn} / \mathrm{Co}$ atomic ordering and valence state. Chem. Mater. 15, 4798-4803 (2003).

35 Guo, H. Z., Gupta, A., Varela, M., Pennycook, S. \& Zhang, J. Local valence and magnetic characteristics of $\mathrm{La}_{2} \mathrm{NiMnO}_{6}$. Phys. Rev. B 79, 172402 (2009).

36 Barón-González, A. J., Frontera, C., García-Muñoz, J. L., Roqueta, J. \& Santiso, J. Magnetic, structural properties and B-site order of two epitaxial $\mathrm{La}_{2} \mathrm{CoMnO}_{6}$ films with perpendicular out-of-plane orientation. J. Phys. Conf. Ser. 200, 092002 (2010).

37 Guo, H. Z., Gupta, A., Zhang, J., Varela, M. \& Pennycook, S. J. Effect of oxygen concentration on the magnetic properties of $\mathrm{La}_{2} \mathrm{CoMnO}_{6}$ thin films. Appl. Phys. Lett. 91, 202509 (2007).

38 Singh, M. P., Truong, K. D. \& Fournier, P. Magnetodielectric effect in double perovskite $\mathrm{La}_{2} \mathrm{CoMnO}_{6}$ thin films. Appl. Phys. Lett. 91, 042504 (2007).

39 Egoavil, R., Hühn, S., Jungbauer, M., Gauquelin, N., Béché, A., Van Tendeloo, G., Verbeeck, J. \& Moshnyaga, V. Phase problem in the B-site ordering of $\mathrm{La}_{2} \mathrm{CoMnO}_{6}$ : impact on structure and magnetism. Nanoscale 7, 9835-9843 (2015).

40 Truong, K. D., Laverdière, J., Singh, M. P., Jandl, S. \& Fournier, P. Impact of Co/Mn cation ordering on phonon anomalies in $\mathrm{La}_{2} \mathrm{CoMnO}_{6}$ double perovskites: Raman spectroscopy. Phys. Rev. B 76, 132413 (2007).

41 Bibes, M., Balcells, L. I., Valencia, S., Fontcuberta, J., Wojcik, M., Jedryka, E. \& Nadolski, S. Nanoscale multiphase separation at $\mathrm{La}_{2 \beta} \mathrm{Ca}_{1 \beta} \mathrm{MnO}_{3} / \mathrm{SrTiO}_{3}$ interfaces. Phys. Rev. Lett. 87, 067210 (2001).

42 de Andrés, A., Rubio, J., Castro, G., Taboada, S., Martínez, J. L. \& Colino, J. M. Structural and magnetic properties of ultrathin epitaxial $\mathrm{La}_{0.7} \mathrm{Ca}_{0.3} \mathrm{MnO}_{3}$ manganite films: strain versus finite size effects. Appl. Phys. Lett. 83, 713 (2003). 
43 Wang, H. S., Wertz, E., Hu, Y. F. \& Li, Q. Role of strain in magnetotransport properties of $\mathrm{Pr}_{0.67} \mathrm{Sr}_{0.33} \mathrm{MnO}_{3}$ thin films. J. Appl. Phys. 87, 7409 (2000).

44 Koster, G., Kropman, B. L., Rijnders, G. J. H. M., Blank, D. H. A. \& Rogalla, H. Quasiideal strontium titanate crystal surfaces through formation of strontium hydroxide. Appl. Phys. Lett. 73, 2920 (1998).

45 Biswas, A., Rossen, P. B., Yang, C.-H., Siemons, W., Jung, M.-H., Yang, I. K., Ramesh, R. \& Jeong, Y. H. Universal Ti-rich termination of atomically flat $\mathrm{SrTiO}_{3}(001)$, (110), and (111) surfaces. Appl. Phys. Lett. 98, 051904 (2011).

46 Brinks, P., Siemons, W., Kleibeuker, J. E., Koster, G., Rijnders, G. \& Huijben, M. Anisotropic electrical transport properties of a two-dimensional electron gas at $\mathrm{SrTiO}_{3}$ $\mathrm{LaAlO}_{3}$ interfaces. Appl. Phys. Lett. 98, 242904 (2011).

47 Blok, J. L., Wan, X., Koster, G., Blank, D. H. A. \& Rijnders, G. Epitaxial oxide growth on polar (111) surfaces. Appl. Phys. Lett. 99, 151917 (2011).

48 Schaffer, B., Grogger, W. \& Kothleitner, G. Automated spatial drift correction for EFTEM image series. Ultramicroscopy 102, 27-36 (2004).

49 Jones, L. Smart Align-a new tool for robust non-rigid registration of scanning microscope data. Adv. Struct. Chem. Imaging 1, 8 (2015).

50 Lucas, G., Burdet, P., Cantoni, M. \& Hebert, C. Multivariate statistical analysis as a tool for the segmentation of 3Dspectral data. Micron 52-53, 49-56 (2013).

51 Egerton, R. F. Electron Energy-Loss Spectroscopy in the Electron Microscope Ch. 4.1 (Springer: New York, NY, USA, 2011)

52 Shabadi, V., Major, M., Komissinskiy, P., Vafaee, M., Radetinac, A., Baghaie Yazdi, M., Donner, W. \& Alff, L. Origin of superstructures in (double) perovskite thin films. J. Appl. Phys. 116, 114901 (2014).

53 Hirone, T., Maeda, S. \& Tsubokawa, I. On the magnetic properties of the system MnSbCrSb. J. Phys. Soc. Jpn 11, 1083-1087 (1956).

54 Singh, M. P., Truong, K. D., Jandl, S. \& Fournier, P. Long-range Ni/Mn structural order in epitaxial double perovskite $\mathrm{La}_{2} \mathrm{NiMnO}_{6}$ thin films. Phys. Rev. B 79, 224421 (2009).

55 Baidya, S. \& Saha-Dasgupta, T. Electronic structure and phonons in $\mathrm{La}_{2} \mathrm{CoMnO}_{6}$ : a ferromagnetic insulator driven by Coulomb-assisted spin-orbit coupling. Phys. Rev. B 84, 035131 (2011)

56 Das, H., Waghmare, U. V., Saha-Dasgupta, T. \& Sarma, D. D. Electronic structure, phonons, and dielectric anomaly in ferromagnetic insulating double pervoskite $\mathrm{La}_{2} \mathrm{NiMnO}_{6}$. Phys. Rev. Lett. 100, 186402 (2008).
$57 \mathrm{Li}, \mathrm{H}$., Salamanca-Riba, L., Ramesh, R. \& Scott, J. H. Ordering in (La,Sr)(Al,Ta) $\mathrm{O}_{3}$ substrates. J. Mater. Res. 18, 1698-1704 (2003).

58 Meneghini, C., Ray, S., Liscio, F., Bardelli, F., Mobilio, S. \& Sarma, D. D. Nature of 'disorder' in the ordered double perovskite $\mathrm{Sr}_{2} \mathrm{FeMoO}_{6}$. Phys. Rev. Lett. 103, 046403 (2009).

59 Singh, V. N. \& Majumdar, P. Antisite domains in double perovskite ferromagnets: impact on magnetotransport and half-metallicity. Europhys. Lett. 94, 47004 (2011).

60 Maclaren, I., Wang, Z. L., Wang, H. S. \& Li, Q. The effects of film-substrate mismatch on $\operatorname{Pr}_{0.7} \mathrm{Ca}_{0.3} \mathrm{MnO}_{3}$ thin films. Philos. Mag. A 82, 1405-1417 (2002).

61 Barilo, S. N., Gatalskaya, V. I., Shiryaev, S. V., Kurochkin, L. A., Ustinovich, S. N., Szymczak, H., Szymczak, R. \& Baran, M. Magnetic behavior of single crystals of the perovskite oxides $\mathrm{LaMn}_{1-x} \mathrm{Co}_{x} \mathrm{O}_{3}$. Phys. Status Solidi A 199, 484-490 (2003).

62 Joseph Joly, V. L., Joy, P. A. \& Date, S. K. Magnetic properties of Co-rich compositions $(x>0.5)$ inthe LaMn ${ }_{1-x} \mathrm{Co}_{x} \mathrm{O}_{3}$ series. J. Phys. Condens. Matter 13, L841-L846 (2001).

63 Yuan, N., Liu, X, Meng, F., Zhou, D. \& Meng, J. First-principles study of Lar $\mathrm{CoMnO}_{6}$ : a promising cathode material for intermediate-temperature solid oxide fuel cells due to intrinsic Co-Mn cation disorder. lonics 21, 1675-1681 (2005).

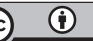

This work is licensed under a Creative Commons Attribution 4.0 International License. The images or other third party material in this article are included in the article's Creative Commons license, unless indicated otherwise in the credit line; if the material is not included under the Creative Commons license, users will need to obtain permission from the license holder to reproduce the material. To view a copy of this license, visit http:// creativecommons.org/licenses/by/4.0/

(C) The Author(s) 2017

Supplementary Information accompanies the paper on the NPG Asia Materials website (http://www.nature.com/am) 